\title{
Central Pleomorphic Adenoma of Mandible - Report of the First Case in a Pediatric Patient
}

\author{
Manas Bajpai* \\ Department of Oral and Maxillofacial Pathology, NIMS Dental College, Jaipur, India.
}

\begin{abstract}
Pleomorphic adenomas (PA) are the commonest salivary gland tumors of parotid gland. Central pleomorphic adenomas are extremely rare and so far only 7 cases have been reported in the literature; 2 in maxilla and 5 in mandible. The mean age of occurrence of central PA is 58.8, none of the case of central PA in children has been reported in the literature; hence this is the first report of central PA in a child. An 11 year old Indian boy presented with the jaw swelling from 1 year. Radiographic examination revealed a multilocular osteolytic lesion of mandible. A complete surgical removal of the lesion was performed and the defect was restored with reconsctruction plates. Histopathological features were consistent with central PA. Till now this is the first description of central PA in a child.
\end{abstract}

Keywords: Central pleomorphic adenoma, Intraosseous salivary gland tumor, Mandible, Pediatric patient, Salivary gland tumor.

\section{INTRODUCTION}

Centrally occurring salivary gland tumors are rare. Because of a considerable overlap between the clinical and histopathological features, this group of tumors often produces a diagnostic difficulty to the clinicians and oral pathologist; moreover these tumors are exceedingly rare in children [1]. Central pleomorphic adenoma (PA) is the rarest possible provisional diagnostic entity that comes to mind, when a patient comes with an osteodestructive lesion of the jaw [2]. An exhaustive literature review reveals only 7 reported cases of this unusual entity; moreover this is the first case of central PA occurring in a child [3]. PA is also called mixed tumor owing to its varied morphology. Clinically Central PA shows painless swelling of jaw with occlusal derangement and subsequent bone destruction, sometimes they imitate the features of benign odontogenic tumors and/or cyst $[4,5]$.

The diagnosis of centrally occurring PA requires a criteria to be fulfilled which include [1]; absence of primary pleomorphic adenoma of major and/or minor salivary gland [2], evidence of osteolysis with integrity of cortical bone on radiographic examination [3], histopathological examination should be consistent with the features of PA [4]. The present case followed these diagnostic criteria [5].

\section{CASE PRESENTATION}

An 11 year old Indian boy was referred to the surgical oncology unit of our institution with the painless swelling on his right lower front region of the face since 1 year. The swelling was initially small but gradually reached the current size. Past medical history and family history of the patient was noncontributory to the presenting symptom. Extra oral

*Address correspondence to this author at the Department of Oral and Maxillofacial Pathology, NIMS Dental College, Jaipur, India.

E-mail: dr.manasbajpai@gmail.com examination revealed a slight deviation of the face towards right side; a bony hard nodule was seen on the right side below the lower lip without any sign of ulceration and discharge (Fig. 1).

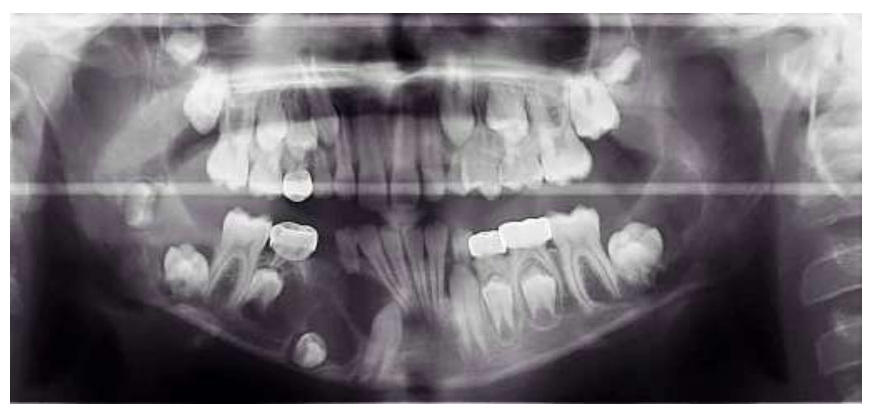

Fig. (1). Clinical Picture of the Lesion.

Intraoral examination revealed buccal and lingual expansion of right mandible with a solitary swelling extending from permanent right mandibular central incisor to right deciduous canine measuring about $3 \times 2 \mathrm{~cm}$. On palpation the swelling was found to be bony hard. The color of the overlying mucosa was similar to the adjacent mucosa. No signs of ulceration and discharge were noted. Parasthesia was not present. Cervical lymph nodes were non palpable.

Panoramic radiograph revealed a large multilocular lesion surrounded by sclerotic margins extending from the root of permanent right mandibular lateral incisor to the right deciduous first molar. Permanent right mandibular canine first and second premolars were erupting (Fig. 2). Based on clinical and radiographic features a provisional diagnosis of ameloblastoma was given.

Hemimandibulectomy was performed under general anesthesia with $1 \mathrm{~cm}$ safety margins by submandibular approach. The defect was restored with $2.4 \mathrm{~cm}$ reconstruction

www.njhsciences.com 
plates. The follow up period of 2 years was uneventful.

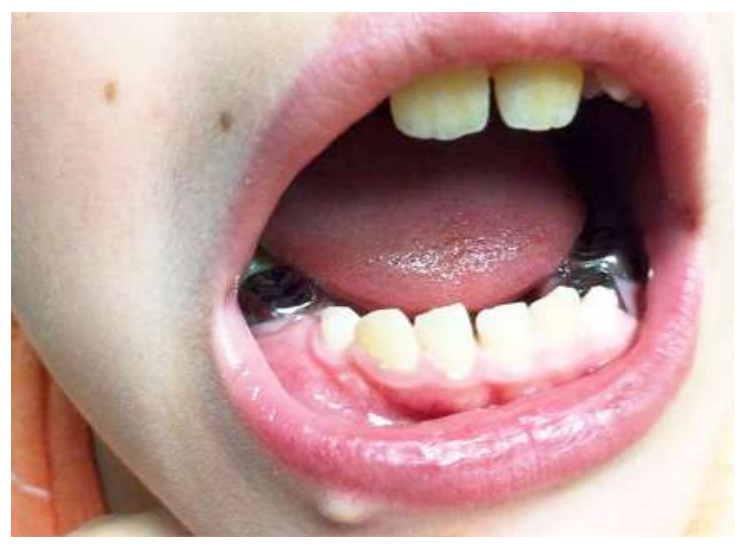

Fig. (2). Panoramic Radiograph Shows a Large Multilocular Osteodestructive Lesion of the Mandible.

The resected specimen was submitted to the Department of Oral and Maxillofacial Pathology, NIMS Dental College for Histopathological Examination. Histopathological examination of hematoxylin and eosin stained soft tissue sections revealed a well encapsulated lesion with proliferation of polygonal and myoepithelial cells in an abundant myxoid connective tissue stroma (Fig. 3). The epithelial component was arranged in a duct like pattern and in sheets form. Based on histopathological features a final diagnosis of central PA was rendered.

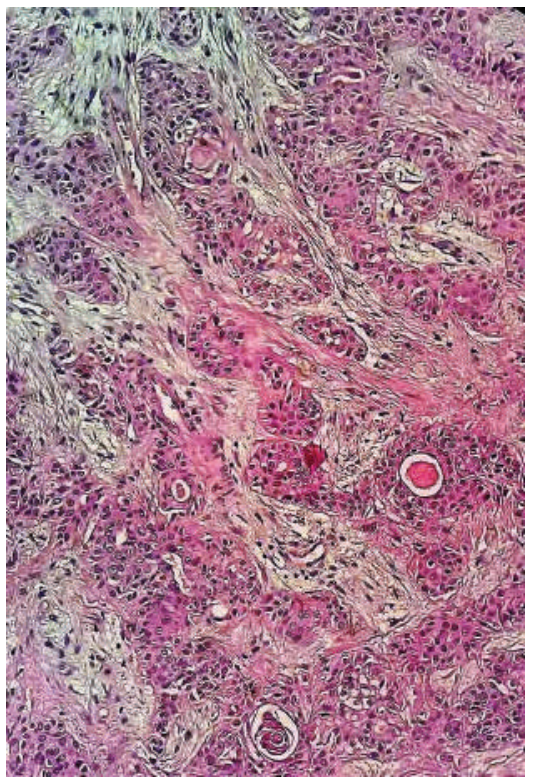

Fig. (3). Arrangement of Epithelial and Myoepithelial Cells in Duct Like Fashion in a Myxoid Connective Tissue Stroma, Few Areas Show Squamous Metaplasia. (Hematoxylin and Eosin Stain X20).

\section{DISCUSSION}

Centrally occurring salivary gland neoplasms are extremely rare [4]. The etiopathogenesis of these neoplasms is not clearly understood, although two different theories have been proposed. First theory suggests that neoplastic transformation in the ectopic glandular tissue of the bone may give rise to the intraosseous salivary gland tumors, the second theory suggests that these tumors may arise from the metaplasia of odontogenic cystic lining i.e. mucoepidermoid carcinoma from dentigerous cystic lining [5-7]. The present case supports the first theory of occurrence as no association of odontogenic cyst was found. The mean age of occurrence of central PA is 58.8 years; however the present case was rare and the first reported case in a child [2]. Females are found to be more commonly affected with the ratio of $1: 3$ to $1: 4$. The most common clinical feature of central PA is swelling with or without pain; in present case also the patient had a painless swelling. Radiographically central PA shows an osteolytic lesion with multilocular or unilocular pattern. The differential diagnoses include ameloblastoma [8], odontogenic keratocyst followed by giant cell granuloma and aneursmal bone cyst [4]

PAs are histopathologically characterized by varied morphology consisting of proliferation of myoepithelial and epithelial cells in ductal architecture with areas of myxoid degeneration, osseous metaplasia, cartilaginous metaplasia and squamous metaplasia. The present case showed squamous metaplasia and abundant myxoid stroma [1,2].

The most common central salivary gland tumors are mucoepidermoid carcinomas [4]. Only 7 cases of central PA have been reported in the literature so far; 5 cases in mandible and 2 cases in maxilla. The occurrence of PA in maxilla is found questionable by some researchers owing to the possibility of their occurrence from the mucous secreting cells of the maxillary sinus [5]. Surgical resection of the jaw is the most preferable mode of treatment with a long term follow up. The present case was treated with the same approach and the follow up period of 2 years was uneventful.

\section{CONCLUSION}

We conclude that a dental surgeon should consider centrally occurring salivary gland tumor as one of the diagnostic possibility for a central jaw lesion. We advocate that; based on their prevalence centrally occurring salivary gland tumors should also be included in the classification of salivary gland tumors. A long term follow up is required after the surgery owing to the paucity of information about central PA regarding their malignant transformation and recurrence.

\section{CONFLICT OF INTEREST}

Declared None. 


\section{ACKNOWLEDGEMENTS}

Declared None.

\section{REFERENCES}

[1] Ojha J, Bhattacharyya I, Islam MN, Manhart S, Cohen DM. Intraosseous pleomorphic adenoma of the mandible: Report of a case and review of the literature. Oral Surg Oral Med Oral Pathol Oral Radiol Endod 2007; 104: e21-6.

DOI: $10.1016 /$ j.tripleo.2006.08.013

[2] Brookstone MS, Huvos AG. Central salivary gland tumors of the maxilla and mandibula: A clinicopathologic study of 11 cases with an analysis of the literature. J Oral Maxillofac Surg 1992; 50: 229-36. DOI: 10.1016/0278-2391(92)90317-S

[3] Waldron CA, Kohn ML. Central mucoepidermoid carcinoma of the jaws: Report of four cases with analysis of the literature and discussion of the relationship to mucoepidermoid sialo-odontogenic, and glandular odontogenic cysts. J Oral Maxillofac Surg 1990; 48: 870.

DOI: $10.1016 / 0278-2391(90) 90349-7$
[4] Bajpai M, Pardhe N, Chandolia B, Arora M. Central acinic cell carcinoma of the mandible simulating as benign odontogenic lesion: A case report. Iran J Med Sci (In press).

[5] Zhao D, He H, Fang M, Liu M, Ji B, He Z. Primary central carcinoma ex pleomorphic adenoma of the mandible: Report of a rare case and literature review. Oral Surg Oral Med Oral Pathol Oral Radiol Endod 2011; 112: e24-8.

DOI: $10.1016 /$ j.tripleo.2011.02.046

[6] Kovalik S. Mixed tumor starting from the jaw bone. Otolaryngol Pol 1966; 20: 563-4.

[7] Ellis GL, Auclaire PL, Gnepp DR. Surgical Pathology of Salivary Gland. Philadelphia: WB Saunders Co. 1991; p. 166.

[8] Bajpai M, Agarwal D, Bhalla A, Kumar M, Garg R, Kumar M. Multilocular unicystic ameloblastoma of mandible. Case Rep Dent 2013; 2013: 835892. DOI: 10.1155/2013/835892 